\title{
Detection of Atrial Fibrillation Using an Earlobe Photoplethysmographic Sensor
}

\author{
Thomas Conroy $^{1}$, Jairo Hernandez Guzman ${ }^{2}$, Burr Hall ${ }^{1}$, Gill Tsouri ${ }^{2}$, Jean-Philippe Couderc ${ }^{1}$ \\ ${ }^{1}$ Heart Research Follow-Up Program, Cardiology Department, University of Rochester Medical \\ Center, University of Rochester, Rochester, NY, United States of America \\ ${ }^{2}$ Electrical and Microelectronics Engineering Department, Rochester Institute of Technology (RIT), \\ Rochester, NY, United States of America
}

\begin{abstract}
Atrial Fibrillation $(A F)$ is the most common cardiac arrhythmia in the world, associated with an increased risk of thromboembolic events and an increased mortality rate. Due to the frequently asymptomatic nature of $A F$, a significant portion of $A F$ is subclinical. To address this issue, we tested the feasibility of detecting AF using photoplethysmographic signal acquired from a noninvasive earlobe photoplethysmographic sensor. Photoplethysmographic recordings were taken from $A F$ patients before and after cardioversion procedures, along with recordings from a healthy control group. This signal was analyzed and multiple parameters of heart rate variability were calculated. The parameter with the highest discriminant capability resulted in a sensitivity and specificity of $90.9 \%$. These results show that using earlobe photoplethysmographic signal is a viable, inexpensive, and non-invasive AF detection method that could be invaluable in detecting subclinical $A F$.
\end{abstract}

\section{Introduction}

AF affects more than 2.2 million people in the United States alone, making it the most common cardiac arrhythmia in the world. [1,2] $\mathrm{AF}$ is associated with diminished quality of life, congestive heart failure, devastating thromboembolic events, [3] and an increased mortality rate. [4] AF frequently manifests itself silently, which causes a significant portion of $\mathrm{AF}$ patients to be undiagnosed, known as subclinical AF. [5] Most studies estimate the rate of subclinical AF to be between $10 \%-40 \%$ of all AF patients. [6] Identifying subclinical AF patients is critical because it enables early detection of $\mathrm{AF}$, therefore shifting the overall paradigm around the management of AF patients.

$\mathrm{AF}$ is defined by the WHO-ISFC task force as "irregular, disorganized, electrical activity of the atria." [7] Current AF detection methods usually require ECG recordings, which are difficult to obtain for long-term continuous monitoring. Current technologies, such as ECG patches, are limited to a couple of weeks use because of the rapidly degrading quality of the electrical properties of the skin electrodes.

In this work, we propose to develop an automatic method for the detection of AF based on a wearable photoplethysmographic (PPG) sensor. [8] A PPG sensor monitors only the hemodynamic profile of the cardiac rhythm, rather than an ECG that directly monitors the electrical activity of the heart. Despite this limitation, PPG sensors are appealing because they only require one small area of skin contact to capture their signal. They can be embedded in earphones or jewelry, and represent a noninvasive method for acquiring long-term continuous cardiac monitoring signal. $[9,10]$

Current ECG-based algorithms for the detection of AF have, at best, an expected sensitivity and specificity on the order of 95\%. [11-12] PPG-based methods for AF detection have been developed and reported by others. Lee at al. [13] reported a technique to detect AF using PPG signal acquired by a smartphone light and camera. Ferranti et al. [14] developed an AF detection method using PPG signal acquired from a wrist worn device. These methods have a similar sensitivity and specificity to the ECG-based methods.

We conducted a clinical study involving patients going through electrical cardioversion to treat AF. [15] We measured the PPG signal continuously in these individuals using a single earlobe PPG sensor (HeartSensor HRS07UE, BINAR Integrated Mobile Systems, Washington, USA). Subsequently, we developed an automatic algorithm for pulse detection, and calculated various HRV parameters to ultimately compare their values during sinus rhythm in healthy people, and in patients during AF (baseline pre-cardioversion) and sinus rhythm (post successful cardioversion). We evaluated and report the discriminant power of the proposed method.

\section{Methods}

\subsection{Study Population}


There were three study cohorts: healthy, AF patients before electrical cardioversion (Pre-CV), and the same AF patients recorded after electrical cardioversion (Post-CV). The AF patients in the Pre-CV/Post-CV groups were scheduled for a cardioversion treatment for $\mathrm{AF}$ independent of this study. All data were recorded from subjects and patients at Strong Memorial Hospital, University of Rochester (Rochester, NY). The study protocol was reviewed and approved by the local Institutional Review Board.

\subsection{Signal Conditioning}

A PPG signal is characterized by successive peaks and valleys representing the systolic and diastolic phases of the cardiac output, respectively. The distance between two adjacent maxima is a close approximation of the RR interval, known as the inter-systolic interval (SS interval).

In some PPG signals, a double peaked signal can be observed. This second peak follows the systolic peak and is caused by pressure equalization processes in the artery. [16] In addition to this second peak, the raw PPG signal is usually affected by various noise components including baseline wandering and transient noise due to sensor movement. Therefore, we implemented specific preprocessing steps for removing these noise and trend components, as well as filtering the second peak.

The PPG signal also includes regular fluctuations due to breathing. To reduce these fluctuations, the 'detrend' function from MATLAB was used to remove linear trends from the PPG signals. [17] Once these respiratory fluctuations were reduced, the data is normalized using the standard score method, aka the Z-Score. [18] Then a bandpass filter is applied with lower and upper cutoff frequencies of $0.5 \mathrm{~Hz}$ to $5.5 \mathrm{~Hz}$. These frequencies correspond to heart rates of $30 \mathrm{BPM}$ to $330 \mathrm{BPM}$, which encompasses the minimum and maximum expected human heart rates. [19] This wide bandwidth is essential as AF rhythm results in a wider bandwidth than sinus rhythm. An example of the signal conditioning results is presented in Figure 1.
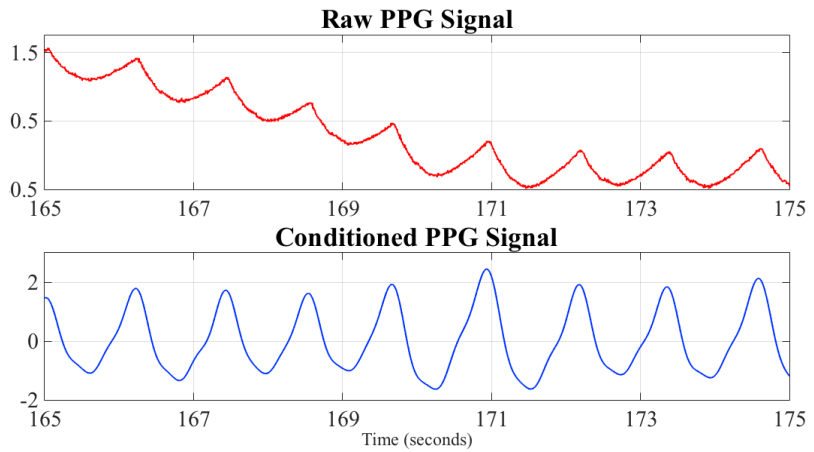

Figure 1. The process of conditioning the PPG signal
In addition to signal conditioning, an algorithm was developed to automatically detect flat signal. This ensured the algorithm could run automatically. This algorithm compared the power of the signal within the bandwidth to the power of the signal outside the bandwidth. [20] If the ratio between these powers indicated low signal power within the bandwidth, the signal was not further processed.

\subsection{Extraction of Pulsatile Signal}

To quantify the irregular irregularity of the heart rate, the pulsatile signal must be extracted from the processed PPG. A cardiac beat is represented by a maximum in the PPG signal; therefore, all maxima were found in the PPG signals, irrelevant of amplitude or proximity to surrounding maxima. This approach alone greatly overestimated the number of cardiac beats.

To address this challenge, a maxima verification algorithm was developed to ensure each maximum appropriately indicated a cardiac beat. This algorithm determined if a maximum is a local maximum, eventually labelling the detected local maxima as cardiac beats. A local maximum was defined as a maximum centered on a window of $2 N$ samples where $N=60$ samples, or 0.2 seconds. [19]

\subsection{Measurements of pulse irregularity}

We calculated four parameters to quantify HRV using on the pulsatile signal. The avg $\Delta \mathrm{SS}$ parameter is the average of absolute value of the differences between each successive SS interval $(\Delta \mathrm{SS})$. [21] The pNNx parameter is a modification of pNN50, a popular HRV measure used in the analysis of heart rhythms for many different cardiac conditions. However, Meitus et al. [22] have shown that for $\mathrm{pNNx}$, using $\mathrm{x}<50 \mathrm{~ms}$ is often more effective at distinguishing between physiological and pathological groups. Therefore, we tested various values of $\mathrm{x}$ for the $\mathrm{pNNx}$ parameter on our learning set to maximize the discrimination between $\mathrm{AF}$ and non-AF rhythm. The SDSS parameter is the standard deviation of SS intervals. The CVSS parameter is calculated by dividing the SDSS by the average SS interval. [18]

It was necessary to set thresholds for the parameters described above to distinguish between Non-AF and AF cohorts. This was done using Receiver Operating Characteristic (ROC) curves. [23] The Non-AF group included the data from healthy subjects and from Post-CV patients after successful cardioversions. The AF group included the Pre-CV data and two recordings from subjects who did not successfully cardiovert to sinus rhythm. Once the thresholds were found for each of the four parameters, the optimal parameter was used to calculate the final accuracy of the proposed method. 


\section{Results}

\subsection{Study Population}

There were 110 total data recordings available in the learning dataset: 46 healthy, 30 Post-CV, and 34 Pre-CV AF recordings. Of these 110 data samples, 3 were found to have flat signals, most likely due to poor device fitting or technical malfunction, and 1 sample was too short (under 4 minutes). Excluding these 4 samples yielded a learning dataset of 106 PPG signals from: 44 healthy subjects, 29 Post-CV, and 33 Pre-CV AF patients.

All subjects included both men and women aged 18 years or older. Healthy subjects $(n=44)$ were $38.1+/-12.4$ years old. Of the healthy group: $58.8 \%$ were female $(\mathrm{n}=$ $25)$ while $43.2 \%$ were male $(n=19), 70.5 \%$ were White $(n$ =31), 29.5\% were African American $(\mathrm{n}=13)$, and 6.8\% were Hispanic or Latino $(\mathrm{n}=3)$. Subjects scheduled for cardioversion $(\mathrm{n}=33)$ were $64.1+/-11.0$ years old. Of the CV group: $84.8 \%$ were male $(\mathrm{n}=28), 15.2 \%$ were female $(\mathrm{n}=5), 100 \%$ were White $(\mathrm{n}=33)$, and $3.0 \%$ were Hispanic or Latino $(\mathrm{n}=1)$.

The validation dataset included 55 PPG signals from: 13 healthy subjects, 21 Post-CV, and 21 Pre-CV AF patients. In the validation dataset, healthy subjects $(n=13)$ were $45.4+/-17.6$ years old. Of the healthy group: $92.3 \%$ were female $(\mathrm{n}=12)$ while $7.7 \%$ were male $(\mathrm{n}=1), 84.6 \%$ were White $(\mathrm{n}=11), 7.7 \%$ were African American $(\mathrm{n}=1)$, and $7.7 \%$ were more than one race $(n=1)$, and $7.7 \%$ were Hispanic or Latino $(\mathrm{n}=1)$. Subjects scheduled for cardioversion $(\mathrm{n}=21)$ were $68.6+/-11.7$ years old. Of the CV group: $76.2 \%$ were male $(\mathrm{n}=16), 23.8 \%$ were female $(\mathrm{n}=5)$, and $100 \%$ were White $(\mathrm{n}=21)$.

\subsection{Pulse Measurement}

The PPG data recorded in this study had a duration of $5.4+/-0.3$ minutes across the study groups. The signal was acquired at a $300 \mathrm{~Hz}$ sampling frequency, resulting in $97,792+/-5634$ samples per recording.

We computed the values of the parameters CVSS, SDSS, avg $\Delta S S$, and the optimal $x$ value for the $p N N x$ parameter on the learning dataset. Eleven values of $\mathrm{x}$ were tested for the $\mathrm{pNNx}$ parameter: from $10 \mathrm{~ms}$ to $60 \mathrm{~ms}$ by steps of $5 \mathrm{~ms}$. This optimization process showed that $\mathrm{x}=$ $35 \mathrm{~ms}$ is associated with the best classification performances.

The pNN35 parameter had the best classification performance on the learning dataset, achieving high sensitivity and specificity. Therefore, we implemented a detection method using the $\mathrm{pNN} 35$ parameter to calculate final sensitivity and specificity results when identifying AF. We summarized the results from the validation step in the Table 1. This table provides: the average and standard deviations of the parameter values for the AF and non-AF recordings, p-values determined by a two-independent sample t-test, and the threshold (THR) computed using ROC curves on the learning dataset. [18] It also provides sensitivity and specificity values for each parameter on the validation dataset.

Table 1. Statistical Analysis of Validation Dataset.

\begin{tabular}{llllll|}
\hline $\begin{array}{l}\text { Parameter } \\
\text { (units) }\end{array}$ & $\begin{array}{l}\text { AF } \\
(\mathrm{n}=22)\end{array}$ & $\begin{array}{l}\text { Non-AF } \\
(\mathrm{n}=33)\end{array}$ & $\begin{array}{l}\text { P- } \\
\text { value }\end{array}$ & THR & Sens Spec \\
\hline $\begin{array}{l}\text { pNN35 } \\
(\%)\end{array}$ & $\begin{array}{l}83.1+/- \\
14.7\end{array}$ & $\begin{array}{l}32.0+/- \\
26.4\end{array}$ & $\begin{array}{l}1.8 * \\
10^{-12}\end{array}$ & 74.3 & 90.990 .9 \\
\hline CVSS & $0.24+/-$ & $0.10+/-$ & $1.3 *$ & 0.17 & 90.981 .8 \\
$(\mathrm{nu})$ & 0.07 & 0.08 & $10^{-8}$ & & \\
\hline SDSS (s.) & $0.19+/-$ & $0.09+/-$ & $1.1 *$ & 0.13 & 90.972 .7 \\
& 0.06 & 0.07 & $10^{-7}$ & & \\
\hline $\begin{array}{l}\text { avg } \Delta S S \\
(\mathrm{~s} .)\end{array}$ & $0.21+/-$ & $\begin{array}{l}0.06+/- \\
0.07\end{array}$ & $\begin{array}{l}2.4 \\
10^{-9}\end{array}$ & 0.14 & 81.887 .9 \\
\hline
\end{tabular}

The area under the pNN35 ROC curve was 0.95. Using pNN35: patients in the healthy cohort were correctly identified a $100 \%$ rate, patients in the Pre-CV cohort were correctly identified at a $90.5 \%$ rate, and patients in the Post-CV cohort were correctly identified at an $85.7 \%$ rate.

\section{Discussion}

These results clearly show that the proposed method enables accurate, automatic detection of AF using a noninvasive earlobe PPG sensor.

As previously discussed, this technology has the potential to shift the current paradigm around the management of AF patients by enabling the detection of AF in subclinical patients. As AF persists without treatment, the likelihood of a stroke event significantly increases; early diagnosis of subclinical AF is the foremost solution to this problem. [3] With an earlobe cardiac monitoring device, continuous cardiac measurements can be taken anytime a patient is wearing the device. These measurements can be used to identify subclinical AF patients earlier than using current monitoring technologies that, in general, require a prescription.

This algorithm could be embedded into wearable devices to provide continuous cardiac monitoring capabilities. One of the key strengths of our method is the limited constraints on the subjects. An earlobe PPG signal can be taken anytime the patient is wearing the device, unlike a PPG acquired by a smartphone camera in which the patient needs to actively read their pulse, or an ECG method that is often administered in a controlled setting with medical personnel. [13]

Our method could be improved upon by using an 
accelerometer to detect excess sensor movement during data recording, as demonstrated by Ferranti et al. [14] Scanning the PPG signal for ventricular premature contractions (VPCs) could also improve our method. The algorithm failed on two recordings in the learning dataset. Both recordings contained large numbers of VPCs that mimicked the pulse irregularity indicative of AF. VPCs have certain telltale characteristics that may allow them to be identified and removed from the PPG signal. [24]

This technology is promising and could be implemented into future wearable devices. AF, while not curable, is treatable yet its combination of an asymptomatic nature and increasing prevalence make it a dangerous condition. The proposed technology can indicate when a person is experiencing an AF episode which has the potential to shift the current paradigm of AF management and treatment.

\section{Acknowledgements}

We would like to thank Dr. Derek DeBusschere from Verily Life Science (Palo Alto, CA, USA) for his support and help in conducting the study described in the paper.

\section{References}

[1] Heeringa J, van der Kuip DA, Hofman A, Kors JA, van HG, Stricker BH, Stijnen T, Lip GY, Witteman JC. Prevalence, incidence, and lifetime risk of atrial fibrillation: the Rotterdam study. Eur Heart J. 2006;27: 949-953

[2] Feinberg WM, Blackshear JL, Laupacis A. Prevalence, age distribution and gender of patients with atrial fibrillation. Analysis and implications. Arch Intern Med. 1995;155: 469473

[3] Wolf PA, Abbott RD, Kannel WB. Atrial Fibrillation as an Independent Risk Factor for Stroke: The Framingham Study. Stroke. 1991;22: 983-988.

[4] Benjamin EJ, Wolf PA, D'Agostino RB, Silbershatz H, Kannel WB, Levy D. Impact of atrial fibrillation on the risk of death: the Framingham Heart Study. Circulation. 1998;98(10): 946-952.

[5] Flaker GC, Belew K, Beckman K, et al. Asymptomatic atrial fibrillation: Demographic features and prognostic information from the Atrial Fibrillation Follow-Up Investigation of Rhythm Management (AFFIRM) study. American Heart Journal. 2005;149(4): 657-663.

[6] Rho RW, Page RL. Asymptomatic Atrial Fibrillation. Progress in Cardiovascular Diseases. 2005;48(2): 79-87

[7] Robles de Medina EO, Bernard R, Coumel P, et al. WHOISFC Task Force. Definition of terms related to cardiac rhythm. Am Heart J. 1978; 95: 796-806.

[8] Allen J. Photoplethysmography and its application in clinical physiological measurement. Physiological Measurement. 2007;28(3).

[9] Poh MZ, Kim K, Goessling A, Swenson N, Picard R. Cardiovascular Monitoring Using Earphones and a Mobile Device. IEEE Pervasive Computing. 2012;11(4): 18-26.

[10] Poh MZ, Swenson N, Picard R. Motion-Tolerant Magnetic Earring Sensor and Wireless Earpiece for Wearable Photoplethysmography. IEEE Transactions on Information
Technology in Biomedicine 2010;14(3): 786-794

[11] Tateno K, Glass L. A method for detection of atrial fibrillation using RR intervals. Computers in Cardiology. 2000;391-394

[12] Couceiro R, Carvalho P, Henriques J, Antunes M, Harris M, Habetha J. Detection of Atrial Fibrillation Using Modelbased ECG Analysis. 19 in $^{\text {th }}$ International Conference on Pattern Recognition. 2008: 1-5.

[13] Lee J, Reyes BA, McManus DD, Mathias O, Chon KH. Atrial fibrillation Detection Using an iPhone 4S. IEEE Transactions on Biomedical Engineering. 2012;60(1): 203206.

[14] Ferranti L, Laureanti R. Atrial Fibrillation detection in PPG signal recorded through a wristband device. Polytechnic University of Milan. 2015.

[15] Guzman JH, Couderc JP, Tsouri GR. Accurate Detection of Cardiac Pulse Using Facial Video Plethysmography. Submitted to IEEE Transactions on Biomedical and Health Informatics.

[16] Elgendi M. On the Analysis of Fingertip Photoplethysmogram Signals. Current Cardiology Reviews. 2012;8(1): 14-25.

[17] Ostchega Y, Porter KS, Hughes J, Dillion CF, Nwankwo T. Resting pulse rate reference data for children, adolescents, and adults: United States, 1999-2008. National health statistics reports. National Center for Health Statistics. 2011;41: 4

[18] Everitt BS, Skrondal A. The Cambridge Dictionary of Statistics. Cambridge University Press. 1998;4: 89

[19] Chhabra L, Goel N, Prajapat L, Spodick DH, Goyal S. Mouse Heart Rate in a Human: Diagnostic Mystery of an Extreme Tachyarrhythmia. Indian Pacing and Electrophysiology Journal. 2012;12(1): 32-35.

[20] Carlson B. Communication Systems: An Introduction to Signals and Noise in Electrical Communication. McGrawHill. 1968;4: 382-383

[21] Huang C, Ye S, Chen H, Li D, He F, Tu Y. A Novel Method for the Detection of the Transition Between Atrial Fibrillation and Sinus Rhythm. IEEE Transactions on Biomedical Engineering. 2011;58(4): 1113-1119.

[22] Meitus JE, Peng C-K, Henry I, Goldsmith RL, Goldberger AL. The pNNx files: re-examining a widely used heart rate variability measure. Heart. 2002;88: 378-380.

[23] Fawcett, Tom. An introduction to ROC analysis. Pattern Recognition Letters. 2006;26(8): 861-874.

[24] Kistin AD, Landowne M. Retrograde Conduction from Premature Ventricular Contractions, a Common Occurrence in the Human Heart. Circulation. 1951;3: 738-751.

Address for correspondence.

Name. Thomas Conroy

Full postal address. 132 Windmill Trail, Rochester, NY 14624

E-mail address. conroytb@clarkson.edu 